\title{
Bone Metabolism In Patients With Type 1 \\ Neurofibromatosis: Key Role of Sun Exposure and Physical Activity
}

\section{Ursula Pia Ferrara \\ "Federico II" University}

Cristina Tortora

"Federico II" University

Carmen Rosano BD

"Federico II" University

Antonia Assunto BD

"Federico II" University

Alessandro Rossi

"Federico II" University

Stefano Pagano

"Federico II" University

Mariateresa Falco

Ospedali Riuniti San Giovanni di Dio e Ruggi d'Aragona

Chiara Simeoli

"Federico II" University

Rosario Ferrigno

"Federico II" University

Alessandra D'Amico

"Federico II" University

Dario Salvio

"Federico II" University

Giuliana Cangemi

Istituto Giannina Gaslini

Rosario Pivonello

"Federico II" University

Pietro Strisciuglio

"Federico II" University

Daniela Melis ( $\nabla$ dmelis@unisa.it)

University of Salerno 


\section{Research Article}

Keywords: Neurofibromatosis type 1, osteopenia, sun, physical activity, vitamin D supplementation.

Posted Date: August 23rd, 2021

DOl: https://doi.org/10.21203/rs.3.rs-787889/v1

License: (c) (i) This work is licensed under a Creative Commons Attribution 4.0 International License. Read Full License

Version of Record: A version of this preprint was published at Scientific Reports on March 14th, 2022. See the published version at https://doi.org/10.1038/s41598-022-07855-4. 


\section{Abstract}

Bone metabolism has been rarely investigated in children affected by Neurofibromatosis (NF1). Aim of the present study was to assess bone mineral metabolism in children and adults NF1 patients, to determine the relevant factors potentially involved in the development of reduced bone mineral density (BMD), and provide possible therapeutic intervention in NF1 patients. 114 NF1 patients and sex and age matched controls were enrolled into the study. Clinical and biochemical factors reflecting bone metabolism were evaluated. Factors potentially affecting BMD were also investigated including: physical activity, sun exposure, vitamin D intake. Whenever the presence of vitamin D deficiency was recorded, cholecalciferol supplementation was started and BMD data obtained during supplementation were compared with previous ones. NF1 patients showed lower Z-scores at Dual-Energy X-ray Absorptiometry (DXA) than controls. Physical activity was significantly reduced in NF1 patients than in controls. Sun exposure was significantly lower in NF1 compared to control subjects. At linear regression analysis vitamin $D$ was the most predictive factor of reduced BMD $(p=0.0001)$. Cholecalciferol supplementation significantly increased BMD z.score $(p=0.000)$. We speculated that a combination of different factors, including reduced sun exposure, possibly associated with reduced serum vitamin $D$ levels, and poor physical activity, concur to the impaired bone status in NF1 patients. We also demonstrated that treatment with vitamin D can be effective in improving BMD in NF1 patients, including children. In conclusion, the findings of the current study are expected to have important implications for the follow-up and prevention of osteopenia/osteoporosis in this common genetic disease.

\section{Introduction}

Neurofibromatosis type 1 (NF1, OMIM \#162200) is one of the most common autosomal dominant disorders with multisystem involvement; affects approximately $1 / 3500$ live births [1]; it is characterized by inter- and intra-familial clinical variability. Major features include cafe-au-lait macules (CALMs), skinfold freckling (SF), Lisch nodules (LN), neurofibromas (NF), typical bone abnormalities, and optic pathway glioma (OPG) [1]. Diagnosis is based on the criteria defined at the National Institute of Health 1988 NF consensus conference [2].

NF1 is caused by heterozygous mutations of the NF1 gene (chromosome 17q11.2) which contains 57 constitutive and 3 alternatively spliced exons [3] and encodes neurofibromin, a protein with tumor suppressor function, ubiquitously expressed and involved in pathways affecting cell growth and development of skeletal, cardiovascular, and nervous systems [4]. Neurofibromin is a member of the GTPase-activating protein gene family and can modulate adenylyl cyclase and protein kinase A (PKA), regulators of osteoblasts and osteoclasts cell function. Decreased expression of neurofibromin is correlated with dose-dependent elevation of intracellular RAS-activity and increasing expression of intracellular signaling pathways (mitogen-activated protein kinase, phosphatidil inositol-3-phosphate kinase), and decreasing expression of c-fos, a crucial regulator in osteoblast differentiation [5]. 
The large majority (90-95\%) of disease-causing NF1 mutations includes intragenic mutations, and less than $10 \%$ is represented by large deletions encompassing the entire NF1 gene and its flanking genomic regions at 17q11.2 [6-11]. Most intragenic mutations are truncating or intragenic copy number changes, a smaller percentage are "in frame" variants [12].

As bone features are concerned, NF1 patients are shorter than healthy subjects: several studies highlight proportionated short stature between $8 \%$ and $15 \%$ of patients affected by NF1, thus suggesting a generalized skeleton bone growth decrease [13-16]. Previous studies, performed in NF1 patients, showed local and general dysregulation in bone resorption and remodeling [14, 17-18], and increased formation of osteoclasts [19]. An increased bone fracture rate was observed in NF1 adults [20] and in NF1 postmenopausal women [21]. Reduced bone mineral density (BMD), osteoporosis and increased fracture risk were also found in young NF1 patients [22]. Previous studies reported low levels of serum 25-hydroxy vitamin D3 (25OHD) and reduced BMD and trabecular bone density in both adults and children NF1 patients [13-22]. Although skeletal involvement in patients with NF1 is well known, and osteoporosis has been reported in adult NF1 patients, there is limited knowledge about bone metabolism in NF1 children [13-19].

The aim of the present study was to assess bone mineral metabolism in children and adults NF1 patients, to determine the relevant factors potentially involved in the development of reduced BMD, and provide possible therapeutic intervention.

\section{Results}

The number of patients at each stage of the study is shown in additional file 1. At each stage patients were excluded either due to data unavailability (bone metabolism study) or unwillingness to start vitamin D treatment (vitamin D follow-up study).

\section{Clinical evaluation}

Short stature was detected in $13 / 114$ patients $(11.4 \%)$, particularly in $2 / 31(6.45 \%)$ with mild phenotype, $3 / 39$ (7.69\%) with moderate phenotype and 8/44 (18.18\%) with severe phenotype. Precocious puberty was detected in two patients with severe phenotype (4.54\%).

\section{Life style}

Physical activity was significantly reduced in NF1 patients than in controls. Particularly both vigorousintensity activity $\left(0.24 \pm 0.2\right.$ hours vs $\left.10.5 \pm 3, p=6.7 \mathrm{E}^{-10}\right)$, moderate-intensity activity $(2.81 \pm 0.2$ vs 6.98 $\pm 2.5, p=0.012)$ and walking $(3.09 \pm 0.2$ vs $7.2 \pm 2.5, p=0.02)$ appeared significantly reduced. The time spent sitting every day during the previous week was higher in patients than in controls ( $51 \pm 8$ vs $32.8 \pm$ $7, p=0.01)$.

Sun exposure was low in 68/114 (59.6\%) patients and in 25/114 (21.9\%) control subjects. Overall, sun exposure was significantly lower in NF1 compared to control subjects (Chi square: 33.8 ; p: 0.00; OR 
Diet calcium intake was low in $46 \%$ and in $55 \%$ of patients and the control group, respectively. No significant difference was observed between patients and controls (Chi square: $0.18 ; p: 0,6733 ;$ OR 0,7). Vitamin D intake was low in $69 \%$ and in $66 \%$ of patients and the control group, respectively. No significant difference was observed between patients and controls (Chi square: 0.12; p: 0.7279; OR 1.38).

\section{Biochemical markers of bone metabolism}

Biochemical parameters were evaluated in 108 patients ( 28 with mild phenotype, 36 with moderate phenotype and 44 with severe phenotype).

NF1 patients showed decreased levels of calcium, calcitonin and 250HD, and increased levels of both OC and CTX compared to controls (Table 2).

Calcitonin serum levels were low in $51 / 108,47.2 \%$ of the total group of patients, $18 / 28(64.2 \%)$ with mild phenotype, $14 / 36$ (38.8\%) with moderate phenotype, 19/44 (44.7\%) with severe phenotype.

Vitamin D serum levels were low in $57 / 108$ patients, $52.7 \%$ of the total group of patients, $10 / 28(35.7 \%)$ with mild phenotype, 18/36 (56.25\%) with moderate phenotype, 29/44 (66.6\%) with severe phenotype.

\section{Hormonal studies}

No significant differences were observed between patients and controls.

\section{Bone mineral density}

BMD was evaluated in 108 patients (28 with mild phenotype, 36 with moderate phenotype and 44 with severe phenotype).

NF1 patients showed lower Z-scores at DXA than controls (Table 2).

Osteopenia was found in 21 patients (19.5\%), particularly in 4/28 (14.2\%) with mild phenotype, 5/36 (14.2\%) with moderate phenotype and $12 / 44(27.2 \%)$ with severe phenotype. Osteoporosis was found in $11 / 108$ (10.18\%) patients: 0/28 with mild phenotype, $4 / 36$ (11.1\%) with moderate phenotype and 7/44 (16\%) with severe phenotype.

\section{Correlation studies}

The DXA Z-scores correlated directly with 250HD serum levels $(r=0.533, p=0.0001) ; 250 \mathrm{HD}$ serum levels also inversely correlated with PTH serum levels $(r=-0.253, p=0.002), \operatorname{ALP}(r=-0.387, p=0.001)$ and directly with calcium serum levels $(r=0.18, p=0.01)$

Moderate-intense physical activity correlated with both alkaline phosphatase $(r=0.25, p=0.007)$ and PTH serum levels $(r=0.19, p=0.004)$; moderate activity correlated with PTH serum levels $(r=0.65, p=0.005)$; 
the time spent sitting every day correlated with calcitonine $(r=0.55, p=0.018)$ and inversely correlated with PTH serum levels $(r=-0.638, p=0.008)$.

At linear regression analysis vitamin $D$ was the most predictive factor of reduced BMD (beta $=0.45, p=$ 0.0001). (Fig. 1)

\section{Data obtained after at least 2 years of vitamin D supplementation}

Fifty-seven patients diagnosed with vitamin $D$ insufficiency were followed for a mean period of $2,4 \pm 0.9$ years, range 2-5 years after starting cholecalciferol supplementation. Z-score after supplementation was significantly higher compared to baseline in NF1 patients $(0.7 \pm 1$ vs $-1.1 \pm 1, p=0.000)$ (Fig. 2). No patients showed osteoporosis after two-years of vitamin D supplementation. Vitamin D serum levels significantly increased after supplementation ( $24 \pm 12$ vs $20 \pm 12, p=0.001)$, Alkaline phosphatase significantly decreased ( $175 \pm 76$ vs $159 \pm 76, p=0.04)$. Calcium ( $10.2 \pm 8.8$ vs $9.4 \pm 1.2, p=0.39)$, phosphorus ( $5.06 \pm 5.4$ vs $4.8 \pm 4.3, p=0.68)$ and calcitonin $(3.3 \pm 2.7$ vs $3 \pm 2.8, p=0.35)$ serum levels were similar in the two groups of patients.

\section{Discussion}

This study confirmed the presence of reduced BMD in NF1 patients, including children. Although osteopenia/osteoporosis have been reported in NF1, pathophysiology and correlations with underlying genetic defect are poorly understood [24-28]. To gather information on the factors that may be implicated, bone metabolism, physical activity, sun exposure, calcium and vitamin D intake, and hormonal status were assessed in a large cohort of 114 NF1 patients.

NF1 patients showed reduced calcium, calcitonin and vitamin D and increased OC and CTX serum levels when compared with age- and sex-matched healthy subjects. The observation of decreased levels of calcitonin, marker of osteoblastic activity and bone neo-formation in NF1 patients, is consistent with a previous report [29]. This finding might be considered as a compensatory mechanism against the increased resorption, which is in turn pointed out by high levels of CTX observed in NF1 patients.

The finding of reduced calcitonin and increased OC and CTX levels suggests the presence of both reduced bone deposition and increased bone remodelling. Impaired bone mineralization in NF1 patients may result from several factors [30].

Reduced BMD might be due to negative effects of neurofibromin mutations on bone growth and structure $[13,18]$. The neurofibromin protein, independent of its Ras GTPase-activating activity, can modulate adenyl cyclase activity and PKA $[3,11]$. Since cAMP and PKA are primary signalling pathways regulating osteoblast and osteoclast cell functions in response to PTH, it is plausible that haploinsufficiency of NF1 in humans can result in both decreased bone formation and/or increased osteoclastic activity and the clinical picture of adynamic bone [28]. 
At the cellular level, osteoblasts showed an increase in proliferation and a decreased ability to differentiate and mineralize in vitro [27]. In addition to an osteoblastic defect, NF1(+/-) mice were found to contain elevated numbers of osteoclasts with increased survival, proliferation, migration, adhesion and resorptive activity [28].

Reduced mineralization [12-26] as well as hypovitaminosis D [29-31] have been reported in NF1 patients; however the mechanism underlying the reduced 250HD in NF1 is unknown. Noteworthy, both dietary calcium and vitamin D intake were normal in NF1 patients, without differences when compared with healthy subjects.

Indeed, the current study demonstrated both reduced vitamin D serum levels in NF1 patients and a significant correlation between vitamin D serum levels and BMD. One possible interpretation is that patients with major skin involvement might avoid sun exposure because of aesthetic embarrassment or discomfort (e.g. high number of neurofibromas may impact clothing choice and decrease outdoor activity levels) [32-34]. Indeed, NF1 patients reported a significant reduction in sun exposure compared to control subjects in the current study. Nonetheless, in vitro studies on skin samples might investigate the presence of a derangement of vitamin D production and metabolism in NF1 patients [35].

Endocrine imbalance may also be involved in bone abnormalities: in the current study osteopenia was found in $10.5 \%(12 / 108)$ of total patients and osteoporosis in $6.48 \%(7 / 108)$.

The anabolic effect of IGF-1 on the bone is well known, regulating bone growth and enhancing osteoblast proliferation $[36,37]$. Recently it has been shown that IGF-1 promotes osteoblastic activity activating mTOR-pathway [38, 39]. IGF-1 affects bone status also acting in a paracrine way, in response to mechanical load, such as during physical activity. An increased IGF-1 expression in osteocytes and osteoblasts in response to mechanical load [40-42] has been reported. Conversely, the absence of mechanical stimuli decreases IGF-1-mediated signaling. This strict connection between bone and muscle is synthesized in "mechanostat theory" [43, 44]. According to this model, muscle and bone respond concertedly to each other's modification and to external factors, creating a "Muscle-Bone Unit". The central element of this model is the regulatory feedback loop between tissue strain and bone architecture $[45,46]$. On the basis of these considerations, the reduced physical activity might also contribute to reduced BMD in NF1. It is noteworthy that the results of the current study demonstrated a significantly reduced physical activity in patients when compared to controls. The detection of a correlation between physical activity and both alkaline phosphatase and PTH serum levels suggest the role of physical activity on bone metabolism.

Based on the results of the current study, it is likely that a combination of different factors, including reduced sun exposure, possibly associated with reduced serum vitamin D levels, and poor physical activity, concur to the impaired bone status in NF1 patients.

In addition, this study demonstrated clearly that treatment with vitamin $D$ can be effective in improving BMD in NF1 patients, including children. Further studies are necessary to investigate the effects of other 
factors influencing BMD in NF1 patients, to further delineate the optimal treatment strategy, and to determine the efficacy and safety of high-dose cholecalciferol in NF1children. Prospective clinical trials to determine whether more aggressive interventions such as bisphosphonates will translate into increased bone mass in NF1 are required.

In conclusion, the findings of the current study are expected to have important implications for the followup and prevention of osteopenia/osteoporosis in this common genetic disease.

\section{Patients And Methods}

\section{Patients}

Patients affected by NF1 and followed at the Department of Translational Medicine, Federico II University of Naples, Pediatric Section, were enrolled in the study after the protocol was discussed with each patient (or legal tutor) and informed consent was obtained. Patients' clinical data were obtained from medical records over the past 20 years.

A multidisciplinary team, including endocrinologists, geneticists and radiologists, evaluated all patients.

The enrollment was carried out according to the following inclusion criteria: (i) clinical diagnosis of NF1 based on recommendations of National Institute of Health [2]; (ii) informed consent expression. The exclusion criteria were: (i) concurrent disorders affecting bone metabolism, i.e., primary hyperparathyroidism, hyperthyroidism, renal or hepatic chronic disease, malabsorption, hypercortisolism, hypogonadism; (ii) previous or ongoing treatments with glucocorticoids, chemotherapies, bisphosphonates or other antiosteoporotic drugs; (iii) previous spine or hip fractures; (iv) surgical correction of scoliosis.

Collected clinical information included familiarity, presence or absence of CALMs, intertriginous SF, LN, cutaneous and subcutaneous NFs, plexiform NFs, spinal NFs, bone abnormalities, OPGs, cardiovascular malformations, endocrine system involvement, developmental delay/intellectual disability, cerebrovascular malformations, and occurrence of other neoplasms.

Patients were divided into three groups according to the severity of the phenotype using the classification proposed by Riccardi [20]. Patients presenting with CALMs, axillary freckling, LN, dermal and/or nodular NFs, and non-progressive scoliosis were classified as "mild", those presenting with plexiform NFs, bone abnormalities, precocious or progressive scoliosis were classified as "moderate", and patients with learning disability/mental retardation, OPG and/or other neoplasms, and/or cerebrovascular disease were classified as "severe".

114 individuals diagnosed with NF1 according to National Institutes of Health criteria (2) were enrolled into the study. 69 patients were females and 44 males. The average age at time of diagnosis was 2.7 years (range $0.3-5$ years), wherease the average age at observation was 11.9 years (range 1-23 years). 
40 patients were children (aged between 1 and 11 years), 34 were in pubertal age (aged between 12 and 16) and 40 were adults (aged between 17 and 23).

Based on the phenotype, 31 patients were classified as "mild" (mean age $12.8 \pm 6$, range 2-22 years), 39 as "moderate" (mean age 12.3 \pm 5.9 , range $2-22$ years), and 44 as "severe" (mean age $12.3 \pm 5.5$, range 1-23 years). Demographic and clinical characteristics are reported in Table 1.

All patients were screened for NF1 and SPRED1 mutations by parallel sequencing of the whole coding region and flanking splice sites ( $\pm 10 \mathrm{bp}$ ). Structural rearrangements were assessed by Multiplex Ligation Probe Amplification analysis using the MRC-Holland P295 probe set. A pathogenic or likely pathogenic NF1 variant was found in 90/114 (78.9\%).

114 age- and sex-matched healthy controls were also enrolled. To minimize potential source of bias, none of the controls had disorders or treatments affecting bone metabolism or BMD.

To evaluate the efficacy of vitamin $D$ therapy in patients with vitamin $D$ insufficiency, 57 patients were followed for a mean period of $2,4 \pm 0.9$ years (range $2-5$ years) after starting $250 \mathrm{H}-\mathrm{D}$ supplementation.

\section{Methods}

All methods were carried out in accordance with relevant guidelines and regulations. All experimental protocols were approved by “Comitato Etico Università Federico II", protocol number 315/18

\section{Clinical evaluation}

Short stature was defined as height less than two standard deviations. Pubertal stage was assessed according to Tanner's pubertal stages; growth velocity was also evaluated.

\section{Life style}

In order to assess the potential contribution of "environmental" factors, physical activity, sun exposure, vitamin $\mathrm{D}$ and calcium intake were recordedduring the enrollment visit.

Physical activity was evaluated by administering the International Physical Activity Questionnaire (IPAQ) [23]. For each patient the time spent experiencing different kind of activities during the previous week was calculated: vigorous-intensity activity (hard physical effort and the patient breathe harder than normal), moderate-intensity activity (moderate physical effort, walking) and the time spent sitting.

Sun exposure was considered low with at least two of the following criteria: no arm and skin exposure during summer months; no sunbathing or holiday in sunny places; no working outdoors. Otherwise, it was deemed sufficient.

Diet calcium intake was defined low with at least two of the following criteria: milk assumption less than $100 \mathrm{~mL}$ a day; less than three yogurts in a week; eating cheese less than two times in a week. Otherwise, 
it was deemed sufficient.

Diet vitamin D intake was evaluated through a questionaire evaluating intake of vitamin D-rich-food as portion per week. These specific food included vitamin D enriched cereals, cheese, fish, lentils. Diet Vitamin D intake was defined low with less than 3 portions assumed in a week. Otherwise it was deemed sufficient.

\section{Biochemical markers of bone metabolism}

Bone metabolism was studied evaluating serum calcium, phosphorus, alkaline phosphatase, parathyroid hormone (PTH), calcitonin, 250HD, C-terminal telopeptide of type I collagen (CTX) and osteocalcin (OC) and urinary calcium/creatinine ratio (UCa/UCr). CTX was measured using a serum cross-laps enzymelinked immunosorbent assay (ELISA) kit (Immunodiagnosticsystems, Frankfurt, Germany) and OC was measured using a Microvue Osteocalcin ELISA kit (Quidel corporation, San Diego, CA, USA). ELISA tests were automated on a DSX system (Dynex technologies-Technogenetics-Milan-Italy) following the manufacturer's instructions. The other markers of bone metabolism were measured using immunoassay with commercially available kits.

Considering the vitamin D levels, NF1 patients were classified according to the Endocrine Society's latest guidelines for vitamin D levels [21]: deficiency was defined by $250 \mathrm{HD}$ less than or equal to $20 \mathrm{ng} / \mathrm{ml}$; insufficiency when it was between 21 and $29 \mathrm{mg} / \mathrm{ml}$; normal if it was more than $30 \mathrm{ng} / \mathrm{ml}$.

Whenever the presence of vitamin D deficiency was recorded, vitamin D supplementation was started and BMD data obtained during vitamin supplementation were compared with previous BMD data. Cholecalciferol was used at a dose of 2,000 UI / day in prepubertal patients and 5,000 UI/day in adolescent.

\section{Hormonal studies}

To address the impact of endocrine regulation on bone homeostasis, the different endocrine axes were evaluated. The somatotropic axis was evaluated by analyzing basal serum growth hormone (GH) and insulin-like growth factor 1 (IGF-1) via immune assay (CLIA); the thyrotropic axis function by analyzing serum thyroid-stimulating hormone (TSH), free triiodothyronine (fT3), free thyroxine (fT4), T3, T4; the adrenocorticotropic axis function by analyzing plasma adrenocorticotropic hormone (ACTH), serum cortisol, androstenedione, 17hydroxyprogesteron (170HP), dehydroepiandrosterone sulphate (DHEA-S), renin, aldosterone levels and 24-hour urinary free cortisol (UFC); the gonadotropic axis function by analyzing serum follicle-stimulating hormone (FSH), luteinizing hormone ( $\mathrm{LH})$, 17 $\beta$-estradiol, testosterone levels. The beta-cell function was analyzed by evaluating basal serum insulin levels, all measured by using immunoassay with commercially available kits.

\section{Bone mineral density}

BMD was studied using Dual-emission X-ray absorptiometry (DXA). DXA (Hologic QDR 1000, Hologic Inc., Waltham, USA). Since both children and adult participants were enrolled, BMD was measured at the L1- 
L4 vertebrae, considering that the hip is not a reliable site for measurement in growing children. Z-scores were calculated by comparing BMD with age and sex matched reference values according to the manufacturer's internal reference database.

Osteopenia was defined as the presence of BMD between - 1.5 and - 2 SD while Osteoporosis was defined as BMD below - 2SD.

\section{Statistical analysis}

All data mentioned in the text or shown in the figure are expressed as mean \pm standard deviation (SD). Sample size calculated using the following formula (with respect to the variable BMD): $(r+1 / r) \times\left[S D^{2}\right.$ $\left.\left(Z_{\beta}-Z_{\alpha / 2}\right)^{2}\right] / d^{2}$ was $108\left(r=1\right.$, SD and $d$ were retrieved from [26], $Z_{\beta}$ was calculated for $80 \%$ power, $Z_{\alpha / 2}$ was calculated at $p<0.05)$ Statistical analysis was performed using Statistical Package for Social Science (SPSS 18) for Windows Update; SPSS Inc., Chicago, Illinois, USA). The comparisons between numerical variables were performed by Student's t test corrected for Fisher's exact test. Pearson's correlation test was performed to assess the relationship among variables with normal distribution ( $p<0.05$ was considered as statistically significant) whereas Spearman's correlation test was performed to assess the relationship among variables with skewed distribution (a $\rho<0.005$ was considered statistically significant). Logistic regression analysis was performed to determine independent predictors of patient outcome (between bone mineral marker and BMD). Univariate analysis of variance and covariance analysis were performed to eliminate the effects of age and gender.

\section{List Of Abbreviations}

25OHD: 25-hydroxy vitamin D3

BMD: Bone Mineral Density

CALMs: cafe-au-lait macules

CTX: C-terminal cross-linking telopeptide of type I collagen

DXA: Dual-Energy X-ray Absorptiometry

LN: Lisch nodules

NF: neurofibromas

NF1: Neurofibromatosis type 1

OC: osteocalcin

OPG: optic pathway glioma

PKA: protein kinase $A$ 
PTH: parathyroid hormone

SF: skinfold freckling

\section{Declarations}

\section{Funding}

No specific funding was received for this study.

\section{References}

1. Ferner, R. Neurofibromatosis 1 and Neurofibromatosis 2: a twenty-first century perspective. Lancet Neurol, 6, 340-351 https://doi.org/10.1016/S1474-4422(07)70075-3 (2007).

2. Stumpf, D. A. J. et al. Neurofibromatosis. Conference statement. National Institutes of HealthConsensus Development Conference. Arch. Neurol., 45, 575-578(1988)

3. Assunto, A. et al. Isoform-specific NF1 mRNA levels correlate with disease severity in Neurofibromatosis type 1. Orphanet J Rare Dis. Nov, 15 (1), 261 https://doi.org/10.1186/s13023-0191223-1 (2019).

4. Kallionpää, R. A. et al. Prevalence of neurofibromatosis type 1 in the Finnish population. Genet Med, 20 (9), 1082-1086 https://doi.org/10.1038/gim.2017.215 (2018).

5. Pasmant, E. et al. Neurofibromatosis type 1 molecular diagnosis: what can NGS do for you when you have a large gene with loss of function mutations? Eur J Hum Genet, 23, 596-601 https://doi.org/10.1038/ejhg.2014.145CrossRef (2015).

6. Upadhyaya, M. et al. An absence of cutaneous neurofibromas associated with a 3-bp inframe deletion in exon 17 of the NF1 gene (c.2970-2972 delAAT): evidence of a clinically significant NF1 genotype-phenotype correlation. Am J Hum Genet, 80 (1), 140-151 (2007).

7. Pinna, V. et al. Et alp.Arg1809Cys substitution in neurofibromin is associated with a distinctive NF1 phenotype without neurofibromas. Eur J Hum Genet;23(8):1068-71(2015)

8. Santoro, C. et al. Arg(1809) substitution in neurofibromin: further evidence of a genotype-phenotype correlation in neurofibromatosis type 1. Eur J Hum Genet;23(11):1460-1(2015)

9. Koczkowska, M. et al. Genotype-Phenotype Correlation in NF1: Evidence for a More Severe Phenotype Associated with Missense Mutations Affecting NF1 Codons 844-848.Am. J Hum Genet, $102(1), 69-87$ (2018).

10. SabbaghA, Pasmant, E., Imbard, A., Luscan, A., Soares, M. \& Blanche', H. et alNF1 molecular characterization and neurofibromatosis type I genotype-phenotype correlation: the French experience. Hum Mutat, 34, 1510-1518 (2013).

11. Trovó-Marqui, A. B. \& Tajara, E. H. Neurofibromin: a general outlook. Clin Genet, 70 (1), 1-13 (2006). 
12. Pasmant, E., Sabbagh, A. \& Spurlock, G. et alNF1 microdeletions in neurofibromatosis type 1: from genotype to phenotype. Hum Mutat, 31, E1506-E1518 (2010).

13. Lammert, M. et al. Decreased bone mineral density in patients with neurofibromatosis 1 . Osteoporos Int, 16, 1161-1166 (2005).

14. Zessis, N. R., Feng Gao, Vadlamudi, G., Gutmann, D. H. \& Hollander, A. S. Height Growth Impairment in Children With Neurofibromatosis Type 1 Is Characterized by Decreased Pubertal Growth Velocity in Both Sexes. J Child Neurol, 33 (12), 762-766 doi: 0.1177/0883073818786807. Epub 2018 Jul 16 (2018).

15. Clementi, M. et al. Neurofibromatosis type 1 growth charts. Am J Med Genet, 87 (4), 317-323 (1999).

16. Szudek, J., Birch, P. \& Friedman, J. M. Growth in North American white children with neurofibromatosis 1 (NF1). J Med Genet, 37 (12), 933-938 (2000).

17. Caffarelli, C. et al. Quantitative ultrasound and dual-energy x-ray absorptiometry in children and adolescents with neurofibromatosis of type 1. J Clin Densitom, 13 (1), 77-83 https://doi.org/10.1016/j.jocd.2009.10.002 (2010).

18. Seitz, S., Schnabel, C. \& Busse, B. et alHigh bone turnover and accumulation of osteoid in patients with neurofibromatosis 1 . Osteoporos Int, 21, 119-127 https://doi.org/10.1007/s00198-009-0933-y (2010).

19. Stevenson, D. A. et al. Multiple increased osteoclast functions in individuals with neurofibromatosis type 1. Am J Med Genet A, 155, 1050-1059 (2011).

20. Riccardi, V. M. Neurofibromatosis: clinical heterogeneity. Curr Prob/ Cancer. Aug, 7 (2), 1-34 (1982).

21. Cianferotti, L. \& Brandi, M. L. Guidance for the diagnosis, prevention and therapy of osteoporosis in Italy. Clin Cases Miner Bone Metab, 9, 170-178 (2012).

22. Ozgur Duman, S. et al. Bone metabolism markers and bone mineral density in children with neurofibromatosis type-1. Brain Dev, 30, 584-588 (2008).

23. Lee, P. H., Macfarlane, D. J. \& Lam, T. H. Stewart SMInt Validity of the International Physical Activity Questionnaire Short Form (IPAQ-SF): a systematic review. J Behav Nutr Phys Act, 8, 115 https://doi.org/10.1186/1479-5868-8-115 (2011).

24. Tucker, T. et al. Bone health and fracture rate in individuals with neurofibromatosis 1 (NF1). J Med Genet, 46, 259-265 (2009).

25. Kuorilehto, T. et al. Decreased bone mineral density and content in neurofibromatosis type 1: lowest local values are located in the load-carrying parts of the body. Osteoporos Int, 16, 928-936 (2005).

26. Brunetti-Pierri, N. et al. Generalized metabolic bone disease in neurofibromatosis type I. Mol Genet Metab, 94, 105-111 (2008).

27. Kolanczyk, M., Kossler, N., Kuhnisch, J., Lavitas, L. \& Stricker, S. Wilkening Uet al. Multiple roles for neurofibromin in skeletal development and growth. Hum Mol Genet, 16, 874-886 (2007).

28. Yang, F. C. et al. Hyperactivation of p21 ras and PI3K cooperate to alter murine and human neurofibromatosis type 1-haploinsufficient osteoclast functions. J Clin Invest, 116, 2880-28891 
(2006).

29. Lammert, M. et al. Vitamin D deficiency associated with number of neurofibromas in neurofibromatosis 1. J Med Genet, 43, 810-813 (2006).

30. Stevenson, D. A. et al. Pediatric 25-hydroxyvitamin D concentrations in neurofibromatosis type 1. J Pediatr Endocrinol Metab, 24 (3-4), 169-174 https://doi.org/10.1515/jpem.2011.092 (2011).

31. Lodish, M. B. et al. Bone mineral density in children and young adults with neurofibromatosis type 1; Endocrine- Related Cancer;19:817 - 25. doi: 10.1530/ERC-12-0293. (2012)

32. Avery, R. A. et al. Orbital/Periorbital Plexiform Neurofibromas in Children with Neurofibromatosis Type 1: Multidisciplinary Recommendations for Care. Ophthalmology, 124, 123-132 https://doi.org/10.1016/j.ophtha.2016.09.020 (2017).

33. Petramala, L. et al. Bone mineral metabolism in patients with neurofibromatosis type 1 (von Recklingausen disease). Arch Dermatol Res, 304, 325-331 (2012).

34. Armstrong, L. et al. The generalized bone phenotype in children with neurofibromatosis 1: a sibling matched case-control study. Am J Med Genet A, 161, 1654-1661 (2013).

35. Filopanti, M. et al. Trabecular Bone Score (TBS) and Bone Metabolism in Patients Affected with Type 1 Neurofibromatosis (NF1. Calcif. Tissue Int, 104, 207-213 (2019).

36. Chihara, K. \& Sugimoto, T. The action of GH/IGF-I/IGFBP in osteoblasts and osteoclasts Horm. Res, 48 (Suppl. 5), 45-49 (1997).

37. Liu, J. et al. IGF-1 as an early marker for low bone mass or osteoporosis in premenopausal and postmenopausal women J. Bone Miner. Metab, 26, 159-164 (2008).

38. Xian, L., Wu, X., Pang, L., Lou, M. \& Rosen, C. J. T. Qiu et alMatrix IGF-1 maintains bone mass by activation of mTOR in mesenchymal stem cells Nat. Med, 18 (7), 1095-1011 (2012).

39. Bilotta, F. L. et al. Insulin and osteocalcin: further evidence for a mutual cross-talk. Endocrine volume, 59, 622-632 (2018).

40. Wang, Y. et al. MM et al., IGF-I receptor is required for the anabolic actions of parathyroid hormone on bone J. Bone Miner. Res, 22, 1329-1133 (2007).

41. Lean, J. M., Jagger, C. J., Chambers, T. J. \& Chow, J. W. Increased insulin-like growth factorl mRNA expression in rat osteocytes in response to mechanical stimulation Am. J. Physiol, 268, 318-327 (1995).

42. Klein-Nulend, J. et al. and transduction in osteocytes Bone, 54 (2), 182-190 https://doi.org/10.1016/j.bone.2012.10.013 (2013).

43. Gross, T. S., Srinivasan, S., Liu, C. C. \& Clemens, T. L. Bain SNoninvasive loading of the murine tibia: an in vivo model for the study of mechano transduction J. Bone Miner. Res, 17, 493-501 (2002).

44. Tahimic, C. G., Wang, Y. \& Bikle, D. D. Anabolic effects of IGF-1 signaling on the skeleton Front. Endocrinol.;4:6. doi: 0.3389/fendo. 2013.00006. (2013)

45. Schoenau, E. From mechanostat theory to development of the "Functional Muscle-Bone-Unit". J. Musculoskelet. Neuronal Interact, 5, 232-238 (2005). 
46. FrostBone, H. M. "Mass" and the "mechanostat": a proposal Anat.Rec., 219 (1):1-9. (1987)

\section{Tables}

Table 1

Demografic and clinical characteristics of the 114 patients with NF1 included in the study. F, females; M, males; NA, not available.

\begin{tabular}{|c|c|c|c|c|}
\hline Feature & $\begin{array}{l}\text { Mild phenotype } \\
(n=31)\end{array}$ & $\begin{array}{l}\text { Moderate } \\
(n=39)\end{array}$ & $\begin{array}{l}\text { Severe } \\
\text { phenotype }(n= \\
44)\end{array}$ & $\begin{array}{l}\text { Whole cohort } \\
(n=114)\end{array}$ \\
\hline \multirow[t]{2}{*}{ Mean age (average) } & 13 years & 12 years & 12.3 years & 11.9 years \\
\hline & (2-22 years) & $\begin{array}{l}(2-22 \\
\text { years })\end{array}$ & (1-23 years) & (1-23 years) \\
\hline No mutation & $N=8$ & $N=8$ & $N=8$ & $N=24$ \\
\hline Gender & $M=12 ; F=19$ & $\begin{array}{l}M=13 ; F= \\
26\end{array}$ & $M=20 ; F=24$ & $M=45 ; F=69$ \\
\hline CALMs & $31(100 \%)$ & $39(100 \%)$ & $46(100 \%)$ & $114(100 \%)$ \\
\hline Lisch nodules & $11(35.4 \%)$ & $20(51 \%)$ & $25(54.4 \%)$ & $56(49.1 \%)$ \\
\hline $\begin{array}{l}\text { Axillary and/or inguinal } \\
\text { freckling }\end{array}$ & $23(74.1 \%)$ & $33(84.0 \%)$ & $33(71.8 \%)$ & $89(78 \%)$ \\
\hline Plexiform neurofibroma & $0(0.0 \%)$ & $6(15.3 \%)$ & $9(19.6 \%)$ & $15(13 \%)$ \\
\hline $\begin{array}{l}\text { Mild non-progressive } \\
\text { scoliosis }\end{array}$ & $12(38.7 \%)$ & $18(46,5 \%)$ & NA & $40(35 \%)$ \\
\hline Progressive scoliosis & $0(0.0 \%)$ & $14(35.9)$ & $25(54.4 \%)$ & 39 (34.2\%) \\
\hline Heart involvement & $4(12.9 \%)$ & $7(17.9 \%)$ & $12(26.1 \%)$ & $23(20.2 \%)$ \\
\hline OPG & $0(0.0 \%)$ & $0(0.0 \%)$ & $27(58.7 \%)$ & $27(23.6 \%)$ \\
\hline Other tumors & $0(0.0 \%)$ & $0(0.0 \%)$ & $17(37.0 \%)$ & $17(14.9 \%)$ \\
\hline $\begin{array}{l}\text { Development delay and/or } \\
\text { cognitive deficit }\end{array}$ & $0(0.0 \%)$ & $0(0.0 \%)$ & $28(60.9 \%)$ & $28(24.5 \%)$ \\
\hline
\end{tabular}


Table 2

Biochemical markers of bone metabolism and bone mineral density in patients and controls

\begin{tabular}{|c|c|c|c|c|}
\hline Phenotype & & Patients & Controls & $\mathbf{p}$ \\
\hline & Calcium mg/dl & $9.6 \pm 0.88$ & $9.9 \pm 0.4$ & 0.009 \\
\hline & Calcitonin pg/ml & $3.4 \pm 2.7$ & $9.6 \pm 2$ & $6.8 \mathrm{E}^{-44}$ \\
\hline & Osteocalcin ng/ml & $121 \pm 15$ & $67 \pm 5$ & $2.5 \mathrm{E}^{-06}$ \\
\hline & CTX ng/ml & $1.64 \pm 0.04$ & $0.42 \pm 0.005$ & $1.08 \mathrm{E}^{-1 c}$ \\
\hline & Vitamin D ng/ml & $21 \pm 7.3$ & $45 \pm 15$ & $7.3 \mathrm{E}^{-22}$ \\
\hline & z-score & $-1.1 \pm 1$ & $0.1 \pm 0.9$ & $1.1 \mathrm{E}^{-06}$ \\
\hline \multirow[t]{3}{*}{ Mild } & Calcitonin pg/ml & $3.5 \pm 2$ & $9.2 \pm 1.4$ & $3 \mathrm{E}^{-05}$ \\
\hline & Vitamin D ng/ml & $19.5 \pm 7$ & $44.3 \pm 12$ & $9.5 \mathrm{E}^{-09}$ \\
\hline & z-score & $-1 \pm 0.8$ & $0.2 \pm 0.9$ & 0.02 \\
\hline \multirow[t]{3}{*}{ Moderate } & Calcitonin pg/ml & $3.15 \pm 2.3$ & $9.3 \pm 2.1$ & $1.1 \mathrm{E}^{-23}$ \\
\hline & Vitamin D ng/ml & $25.6 \pm 17$ & $47.4 \pm 15$ & $7.3 \mathrm{E}^{-08}$ \\
\hline & z-score & $-0.9 \pm 1.2$ & $0.04 \pm 0.9$ & 0.0005 \\
\hline \multirow[t]{3}{*}{ Severe } & Calcitonin pg/ml & $4.8 \pm 3$ & $10 \pm 2$ & $2 \mathrm{E}^{-07}$ \\
\hline & Vitamin D ng/ml & $15.2 \pm 6$ & $43.9 \pm 15$ & $1.6 \mathrm{E}^{-15}$ \\
\hline & z-score & $-0.6 \pm 1.4$ & $0.16 \pm 0.9$ & 0.01 \\
\hline
\end{tabular}




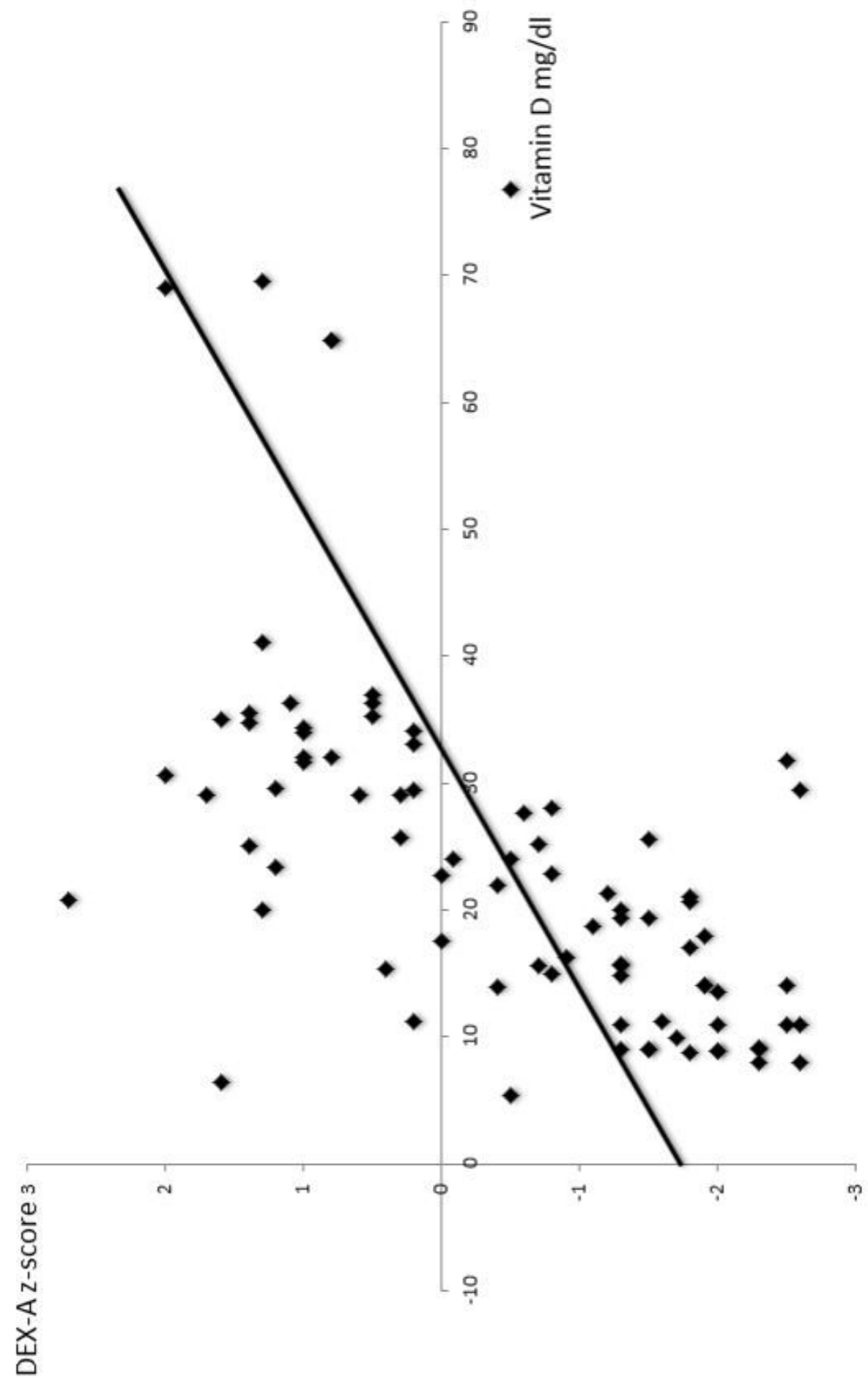

Figure 1

Correlation between vitamin D serum levels and Bone Mineral Density values 


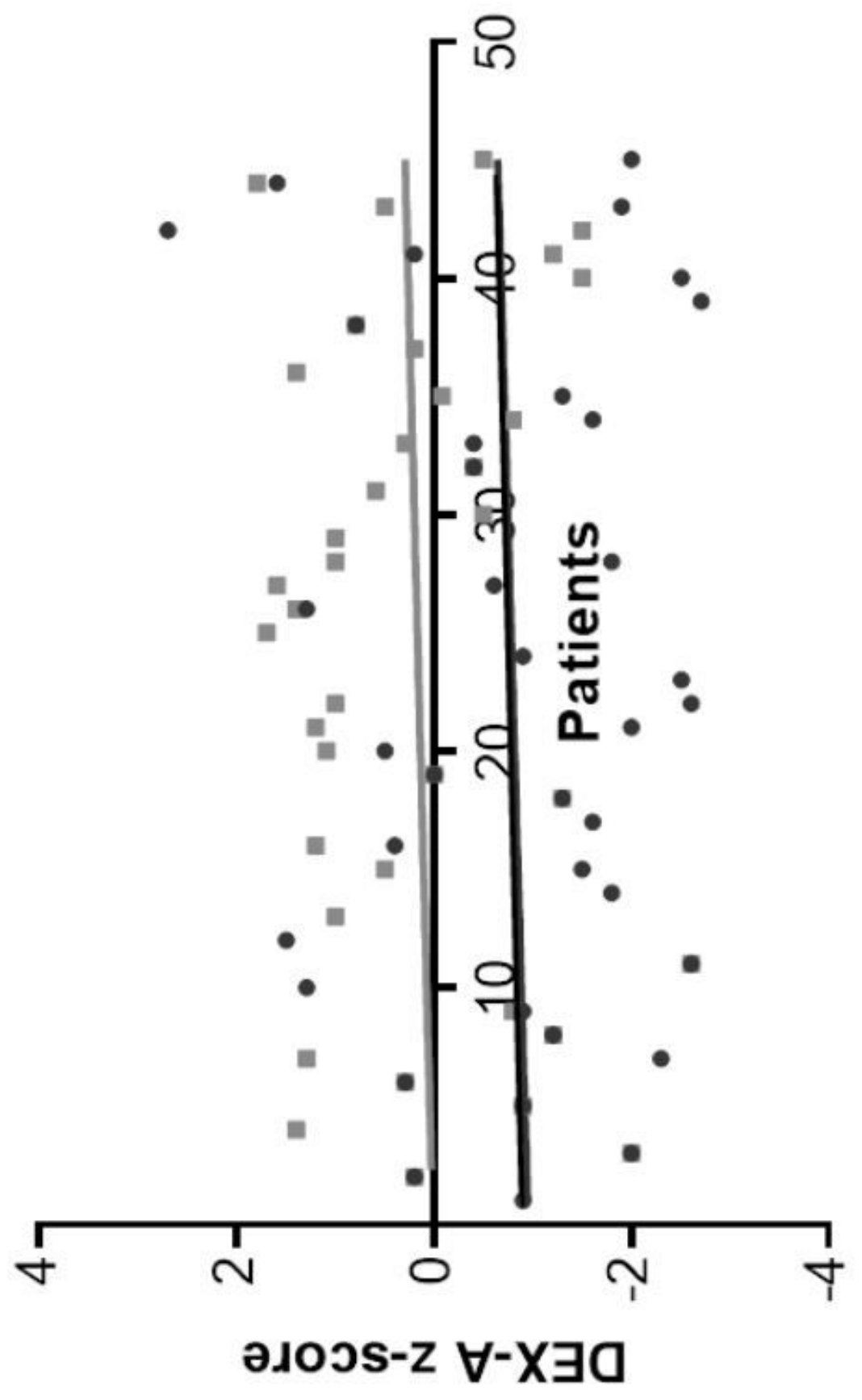

Figure 2

Bone Mineral Density data in patients at baseline (black) and after at least two years of cholecalciferol supplementation (grey) 